\title{
The medical genetics workforce: An analysis of clinical geneticist subgroups
}

Judith A. Cooksey, MD, MPH ${ }^{1}$, Gaetano Forte, BA ${ }^{2}$, Patricia A. Flanagan, MA, MSW ${ }^{1}$, Judith Benkendorf, MS, CGC ${ }^{3}$, Miriam G. Blitzer, $P h D^{1,4}$

\begin{abstract}
Purpose: Clinical geneticists with a Doctor of Medicine degree face challenges to meet the growing population demand for genetic services. This study was designed to assist the profession with workforce planning by identifying clinically relevant subgroups of geneticists and describing their professional characteristics and clinical practices. Geneticists' patient care productivity is compared across subgroups and other medical specialists. Methods: Part of a comprehensive national study of genetic services and the health workforce, this study uses data from a 2003 survey of geneticists certified by the American Board of Medical Genetics. This study includes 610 clinical geneticists who spend at least $5 \%$ of their time in direct patient-care services. An iterative approach was used to identify five subgroups based on the types of new patients seen. We conducted a descriptive analysis of subgroups by demographic, training, professional, and practice characteristics. Results: The subgroups include general (36\%), pediatric (28\%), reproductive (15\%), metabolic (14\%), and adult (7\%) geneticists. Clinically relevant variations across subgroups were noted in training, professional, and practice parameters. Subgroups vary across patient care hours (median, 15-33 hours/week) and total weekly work hours (52-60 hours). New patient visits (mean, 222-900/year) are higher than follow-up patient visits (mean, 155-405) for all subgroups except metabolic geneticists. Conclusion: Although many geneticists practice as generalist geneticists, this study provides an evidence base for distinguishing clinically relevant subgroups of geneticists. Geneticists provide similar numbers of new patient visits and far fewer follow-up visits than other medical specialists. These findings are relevant to geneticist workforce planning. Genet Med 2006:8(10):603-614.
\end{abstract}

Medical geneticists provide comprehensive genetic services to growing numbers of individuals and families. They lead efforts in genetics-related patient care, research, teaching, and clinical laboratory services, and are interpreters of human genome research for professional colleagues and the public. However, those in the profession anticipate changes in workforce-related supply and demand, particularly among clinical geneticists with a Doctor of Medicine (MD) degree, the group most directly involved with patient care.

MD clinical geneticists comprise the primary medical specialist group trained and certified in clinical genetics, although their numbers are small when compared with other medical specialties. In 2005, there were only 1,178 board-certified MD clinical geneticists among the more than 697,000 board-certified physicians. ${ }^{1,2}$

From the ${ }^{1}$ Genetics Health Services Research Center, Department of Epidemiology and Preventive Medicine, University of Maryland School of Medicine, Baltimore, Maryland; ${ }^{2}$ Center for Health Workforce Studies, University at Albany, SUNY, School of Public Health, Albany, New York; ${ }^{3}$ American College of Medical Genetics, Bethesda, Maryland; ${ }^{4}$ Department of Pediatrics, University of Maryland School of Medicine, Baltimore, Maryland.

Judith A. Cooksey, MD, MPH, Genetics Health Services Research Center, Department of Epidemiology and Preventive Medicine, University of Maryland School of Medicine, 660 West Redwood St. Baltimore, MD 21201.

Submitted for publication February 16, 2006.

Accepted for publication August 8, 2006.

DOI: 10.1097/01.gim.0000242307.83900.77
A comprehensive medical genetics workforce study reported the aggregate supply of MD clinical geneticists to be approximately 3.5 geneticists per million population. ${ }^{3}$ There is geographic maldistribution of geneticists across the United States with some states seeming to have an insufficient supply to meet population needs for genetic services. ${ }^{3}$ In addition, MD clinical genetics training programs have seen declining numbers of trainees, a situation leading to fewer entrants into the profession in future years. ${ }^{4}$

A further constraint to meeting population needs for genetic services is the modest number of patients seen by geneticists. For example, the "typical" clinical geneticist works at an academic medical center (AMC), spends approximately $50 \%$ of his or her time in direct patient care, and provides approximately 700 total patient visits per year (380 new patient visits and 320 follow-up visits). ${ }^{5}$ This patient visit volume is substantially smaller than that reported by physicians practicing in other specialties, although the data on other physician specialties include physicians who spend approximately $90 \%$ of their workweek with patient care activities. ${ }^{6}$

To address these and other issues, leaders from medical genetics organizations sponsored a 2004 genetics summit. ${ }^{4}$ Various approaches to the challenges facing clinical geneticists were discussed, including attracting new entrants to the profession, revising genetics training and the certifying examination, and preparing new geneticists for evolving genetics 
practice. In 2006, the American College of Medical Genetics (ACMG) sponsored a follow-up meeting.

There is consensus that clinical applications from genomics research will expand the number of individuals and the scope of medical conditions requiring genetic evaluations. However, the effects of the growing demand for various types of genetic services on clinical geneticists' practices and the workforce are critical unknowns. The purpose of this study is to assess and describe current differentiation in clinical geneticists' practices on the basis of the types of referred patients and other factors.

We designed this study as a follow-up to an extensive survey of all board-certified medical geneticists. ${ }^{3}$ With a two-step process, we sought to identify clinically relevant subgroups of geneticists and to assess the subgroups on their genetics practices, patient care productivity, and other characteristics. The findings are discussed as they relate to the overall clinical geneticist workforce and the utility of considering geneticist subgroups in planning efforts to meet population genetic services needs.

Throughout the article, we use the term "subgroup" rather than "subspecialist" or "subspecialty" because the American Board of Medical Genetics (ABMG) has not yet defined any clinical subspecialties for genetics. However, the ABMG expects to submit a proposal in 2006 to establish a clinical subspecialty of medical biochemical genetics (Karla Matteson, $\mathrm{PhD}$, personal communication, January 2006).

\section{MATERIALS AND METHODS}

This article uses data from a comprehensive national health services research study of genetic service models and the health workforce. ${ }^{7}$ The national genetics study adapted an innovative mixed methods (quantitative and qualitative) research design that was developed by health services researchers to study change in the overall U.S. health care system. ${ }^{8}$ In the genetics study, the quantitative data collection included surveys of genetics providers; and the qualitative data included more than 230 interviews with national experts and genetics providers drawn from in-depth case studies of genetic services in four geographically diverse metropolitan areas of the United States. ${ }^{7}$

There were 31 practicing clinical geneticists among the 230 interviews. They were drawn from 4 of the 12 metropolitan areas representing four different regions of the country. ${ }^{8}$ The geneticist interview sample included all identified practicing geneticists in each area with interviewees selected through a modified, stratified sample process to ensure inclusion of geneticists from various work settings and serving a variety of types of patients. All interviews were conducted by trained interviewers, transcribed, entered into a database, and managed using software designed for qualitative research. ${ }^{7}$ Findings from these interviews provide context to survey findings and are presented as comments in the discussion.

\section{Survey of American Board of Medical Genetics-certified geneticists}

This study's survey data come from a 2003 written survey of all living ABMG-certified medical geneticists (certified before
2002). ${ }^{3}$ The survey (available on request) contained 67 questions covering current status, demographics, education and training, professional practice, genetics patient care activities, clinical genetics laboratory services, and attitudes and professional perspectives.

The survey was sent to 1,567 ABMG diplomates, and responses were received from 861 geneticists (response rate $=54.5 \%$ ). For the analysis of potential response bias, we excluded all geneticists residing outside of the United States (109 in the population and 47 in the respondent sample). Response rate differences were examined by geographic region and date and type of first ABMG certificate (using analysis of variance and multiple comparison techniques). We found no evidence of response bias, indicating that the respondents can be considered representative of the population of board-certified medical geneticists in the United States.

Because population estimates are most important in health workforce studies and planning, we derived weighted estimates to the geneticist population (using census regions and the year and type of $A B M G$ certificate, and then applying a multidimensional simultaneous cross-tabs methodology). Following the weighting, we eliminated any geneticists who were retired or otherwise not professionally active from the analysis. All results are expressed as weighted estimates unless indicated.

Thus for the overall survey analysis, there were an estimated 1,377 active geneticists (certified before 2002) residing in the United States. ${ }^{3}$ Further analysis showed this included MDtrained geneticists (926 geneticists with an MD or equivalent degree, some also possessing a $\mathrm{PhD}$ ) and $451 \mathrm{PhD}$-only trained geneticists. ${ }^{3}$ In the initial survey analysis, we found that approximately $70 \%$ of all MD geneticists reported providing some direct face-to-face genetics patient care. ${ }^{5}$

For this study, we selected a subset of the total geneticists who met three criteria: provision of direct face-to-face patient care (676 geneticists), physicians (as opposed to PhD-only patient care geneticists) (641 geneticists), and reporting that patient care accounts for more than $5 \%$ of their total professional work time (610 geneticists). The weighted sample size for this study was 610, with an actual respondent sample size of 315 geneticists. This sample was considered of sufficient size to allow for the analysis of several subgroups.

\section{Identifying clinical subgroups}

The purpose of this process was to identify a modest number of subgroups of clinical geneticists who had clinical and workforce relevance and had a sufficient sample size to allow descriptive analysis. This goal was particularly challenging because certain characteristics were common across all clinical geneticists. For example, in aggregate $75 \%$ of all MD geneticists were trained in pediatrics, $76 \%$ of all new patients for geneticists were infants and children, and $62 \%$ of all geneticists worked at an AMC. ${ }^{5}$ All of the geneticists were board certified by the ABMG in one or more areas; almost all (98\%) were board certified in MD clinical genetics, the remaining $2 \%$ were board certified in a clinical genetics laboratory area. 
Table 1

Clinical geneticist subgroups: Criteria and size

\begin{tabular}{|c|c|c|c|c|}
\hline Subgroup & Criteria & $\begin{array}{l}\text { Weighted } \\
\text { sample size }\end{array}$ & $\begin{array}{l}\text { Actual } \\
\text { respondent sample size }\end{array}$ & $\begin{array}{l}\text { Percentage } \\
\text { of sample }\end{array}$ \\
\hline Metabolic geneticists & $>40 \%$ of patients with metabolic conditions & 83 & 45 & $14 \%$ \\
\hline Pediatric geneticists & $>80 \%$ of patients are infants or children & 170 & 86 & $28 \%$ \\
\hline Reproductive geneticists & $\begin{array}{l}>60 \% \text { of patients seen for reproductive } \\
\text { genetics consultations }\end{array}$ & 94 & 48 & $15 \%$ \\
\hline Adult geneticists & $\begin{array}{l}>40 \% \text { of patients are adults, many with } \\
\text { adult-onset genetic conditions }\end{array}$ & 42 & 22 & $7 \%$ \\
\hline General geneticists & $\begin{array}{l}\text { Either criteria: general geneticist with }>20 \% \\
\text { of patients from each: infants/children, } \\
\text { reproductive, and adult, or not classifiable } \\
\text { in any other subgroup }\end{array}$ & 222 & 113 & $36 \%$ \\
\hline Total geneticists & & 610 & 315 & $100 \%$ \\
\hline
\end{tabular}

By using the process described next, each geneticist was assigned to one of five subgroups who met specific criteria. The five clinical subgroups include general geneticists (36\% of all geneticists), pediatric geneticists $(28 \%)$, reproductive geneticists (15\%), metabolic geneticists (14\%), and adult geneticists (10\%) (Table 1).

To identify these subgroups, we used an iterative approach that involved examining how potential subgroups met certain patient mix criteria. Responses to the following two survey questions asking about new genetics patients were analyzed. For each question, the total mix of patients should equal approximately $100 \%$.

1. "Estimate the percentage of your new patients from 2002 representing the following groups: (1) newborns and infants ( $<1$ year of age); (2) children and adolescents (not pregnant); (3) prenatal/reproductive care or counseling; and (4) adults (not pregnant)."

2. "Indicate the percentage of your new patients from 2002 with genetic disorders belonging to the following categories: (1) dysmorphologies/syndromes/birth defects; (2) developmental delay/mental retardation; (3) metabolic conditions; (4) reproductive genetics/teratology; (5) cancer; (6) common/complex disorders; (7) adult-onset single gene disorders; and (8) other."

These two questions ask for overlapping information, thus allowing for some comparison of response consistency, which was present. Results from these questions were examined independently from two perspectives. The first perspective was the dominant patient type(s), because this would indicate the major patient focus of the geneticist's practice. We evaluated several cutoff points for the criterion for dominant patient type, for example, $20 \%, 40 \%, 60 \%$, or $80 \%$ of all new patients, and then examined the size and characteristics of the resulting groups.

With these two questions, each categorizing $100 \%$ of patients, it is possible to have several combinations of dominant patient types, depending on the criteria cutoff point. For example, the number of geneticists seeing $40 \%$ or more of all new patients from one or more specified genetic patient or condition categories is shown in Table 2. These data show that some

Table 2

Geneticist subgroups and patient mix: Geneticists with more than $40 \%$ of new patients in specified patient or condition category

\begin{tabular}{|c|c|c|c|c|c|c|}
\hline & All & General & Pediatric & Metabolic & Reproductive & Adult \\
\hline \multicolumn{7}{|l|}{ Patient category } \\
\hline Newborns and infants & $31 \%$ & $21 \%$ & $60 \%$ & $45 \%$ & $0 \%$ & $2 \%$ \\
\hline Children and adolescents & 38 & 27 & 70 & 59 & 0 & 4 \\
\hline Reproductive patients & 20 & 5 & 7 & 4 & 100 & 0 \\
\hline Adults & 10 & 0 & 4 & 13 & 0 & 98 \\
\hline \multicolumn{7}{|l|}{ Genetic condition } \\
\hline Dysmorphology, syndrome, birth defect & 32 & 30 & 67 & 14 & 2 & 4 \\
\hline $\begin{array}{l}\text { Developmental delay, mental } \\
\text { retardation }\end{array}$ & 28 & 28 & 55 & 19 & 2 & 0 \\
\hline Metabolic conditions & 13 & 0 & 0 & 100 & 0 & 0 \\
\hline Cancer & 3 & 0 & 0 & 0 & 0 & 44 \\
\hline Adult-onset single gene & 4 & 0 & 1 & 2 & 2 & 40 \\
\hline
\end{tabular}


geneticists had no dominant patient type in their practice and that some had more than one dominant patient type using a $40 \%$ or more cutoff point. The final cutoff point for the dominant patient type criterion varied by subgroup and was based on iterative analysis of survey data to find the best subgroup differentiation.

A second perspective from these patient mix data allowed us to assess the diversity of patients and genetic conditions seen by geneticists in each subgroup. We selected $10 \%$ of new patients as representing a cutoff point that indicated a commitment to one or more patient or category types, and not simply an occasional patient referral. With this criterion, many geneticists see at least $10 \%$ of new patients from several patient or condition categories (Table 3 ).

We examined responses to various training and professional practice questions across potential subgroups. This allowed us to see whether there were common patterns within the subgroups and to assess how subgroups differed on key variables. This process was repeated several times to identify the final subgroups.

With this information, two subgroups were readily identified. Reproductive geneticists and adult geneticists had clearly defined dominant patient types: reproductive patients or adult genetics patients (or genetic conditions that manifest in adulthood), respectively (Table 2). The final criterion for the adult geneticist subgroup was set relatively low ( $40 \%$ of more of new patients being adult genetics patients) to maximize the size of this small but clinically relevant subgroup. The final criterion for the reproductive geneticist subgroup was set higher $(60 \%$ or more of all new patients being reproductive genetics patients) because this subgroup was larger and the more restrictive criterion allowed for a more tightly defined subgroup.

As expected, many geneticists see large numbers of infants and children (infants/newborns and children/adolescents). Many of these "pediatric" geneticists also see substantial numbers of individuals referred for one or more conditions such as dysmorphologies/syndromes/birth defects, developmental delay/mental retardation, or metabolic conditions. With further analysis, we concluded that most pediatric geneticists saw large numbers of patients with dysmorphologies/syndromes/birth defects or developmental delay/mental retardation and that there were not sufficient differences in other characteristics to further subclassify the subgroup we identified as pediatric geneticists. We set the criterion for pediatric patients high $(80 \%$ of new patients) for this subgroup because it was a large subgroup and the higher, more restrictive cutoff would maximize the likelihood of a more homogenous and distinct subgroup.

Metabolic geneticists were considered a clinically relevant group, and we examined the entire geneticist sample for evidence of a subgroup with a focus on metabolic genetic conditions. This analysis revealed a distinct subgroup of geneticists who saw a large number of metabolic genetics patients. The metabolic geneticist subgroup had other noteworthy characteristics (e.g., training in biochemical genetics and seeing both children and adult genetics patients) that supported a distinct subgroup classification. The final criterion for the metabolic geneticist subgroup required seeing $40 \%$ or more new patients for evaluation of a genetic metabolic condition.

The last of the five defined subgroups was generalist geneticists. This group was initially defined as geneticists who had a general genetics practice, seeing at least $20 \%$ of new patients from each of the major patient categories (infants and/or children, reproductive, and adults). However, after this fifth subgroup was identified, only $75 \%$ of the study sample of geneticists had been classified into a subgroup; the remaining 25\% were unclassified. Various efforts to find any distinct subgroups within the unclassified group were not successful. Although, when we compared this unclassified group with the other five subgroups across professional and practice characteristics, we found many similarities between it and the generalist geneticist subgroup. This led us to consider the unclassified group, which lacked any discernible dominant patient or

Table 3

Geneticist subgroups and patient mix: Geneticists with more than $10 \%$ of new patients in specified patient or condition category

\begin{tabular}{|c|c|c|c|c|c|c|}
\hline & All & General & Pediatric & Metabolic & Reproductive & Adult \\
\hline \multicolumn{7}{|l|}{ Patient category } \\
\hline Newborns and infants & $73 \%$ & $95 \%$ & $84 \%$ & $75 \%$ & $21 \%$ & $21 \%$ \\
\hline Children and adolescents & 79 & 98 & 91 & 89 & 20 & 42 \\
\hline Reproductive patients & 43 & 44 & 28 & 15 & 100 & 21 \\
\hline Adults & 48 & 57 & 29 & 53 & 34 & 100 \\
\hline \multicolumn{7}{|l|}{ Genetic condition } \\
\hline Dysmorphology, syndrome, birth defect & 76 & 95 & 99 & 54 & 33 & 30 \\
\hline Developmental delay, mental retardation & 74 & 96 & 91 & 72 & 22 & 25 \\
\hline Metabolic conditions & 30 & 22 & 27 & 100 & 2 & 7 \\
\hline Cancer & 19 & 27 & 4 & 7 & 16 & 73 \\
\hline Common/complex genetic disorders & 11 & 9 & 7 & 10 & 15 & 39 \\
\hline Adult-onset single gene disorders & 20 & 23 & 12 & 9 & 4 & 90 \\
\hline
\end{tabular}


condition types, as another type of generalist geneticists. Thus, the final generalist geneticist subgroup is the largest subgroup and has two criteria (identifiable diversity of patients or unclassified by any dominant patient type) (Table 1).

\section{Data variables and analysis}

All survey analyses were carried out using the Statistical Package for the Social Sciences software. ${ }^{9}$ The institutional review boards of the University of Maryland and the New York State Department of Health approved the research protocol.

Most survey data variables are self-explanatory. For survey questions that asked respondents to select categoric responses containing numeric values (e.g., from a range of percentage point values, such as " $21 \%$ to $40 \%$ "), we used the midpoint value and present grouped mean or median values. Some survey questions had categoric responses that allowed an ordered subjective response (e.g., "few patients" or "many patients"); therefore, the categories could be combined and we present the number of respondents.

In several data tables, responses are aggregated to simplify reporting. For example, the 11 response categories for primary work setting were grouped into four categories: AMC, physician practice (includes single or multiple specialty medical practice, managed care organization or health maintenance organization), hospital (includes non-AMC hospital, tertiary care public hospital, or children's hospital), and other setting (commercial laboratory, pharmaceutical or biotechnology company, government agency, U.S. military, consulting group, or other).

For the analysis of professional income, geneticists were asked to estimate their net income for 2002 for all professional work activities, after work expenses and before taxes. There were nine response categories, from less than $\$ 50,000$ to greater than $\$ 400,000$.

We designed the survey to assess in detail the patient care productivity of geneticists. Responses to key survey questions (hours worked per week worked per year, number of new and follow-up patient visits, time spent with new and follow-up patients) required specific numeric values, rather than categoric responses. These data allow for more precise estimates of key descriptive data. New variables were derived by calculation using individual responses to more than one survey question. For example, the variable "annual patient care productivity" is defined as the patient visits per year produced by a geneticist. It is calculated by multiplying individual responses for total weekly new and follow-up patient visits with the total weeks worked in a year. The variable "patient care hours per week" used the response for total weekly work hours multiplied by the response for percentage of time spent in patient care.

We also used this variable (patient care hours per week) to identify a special category of geneticists: those who work 20 hours or more in patient care per week. We selected the 20 hour cutoff to allow for the comparison with other physician survey studies conducted by the American Medical Association (AMA). The AMA reports on physician practice patterns and patient care productivity for a limited number of physician specialties, but these surveys include only physicians who work 20 hours or more per week in patient care.6,10

For comparisons with AMA survey findings we note a data limitation of our survey. The AMA surveys ask about various settings for new and follow-up patient visits (e.g., office visits, hospital visits, emergency department visits, and visits in other settings). Our survey simply asked for the number of genetics patient visits in an average week. We do not know whether individual geneticists responded for their office/clinic visits alone or their total visits (hospital visits and clinic/outpatient visits). To assist with the comparison with other medical specialties, we list both the total patient visits and the total office visits.

Because this study was designed to identify clinically relevant subgroups of geneticists and to describe their characteristics, it was not our intent to conduct extensive statistical tests for differences between subgroups. Rather, the study findings are presented as an overall descriptive portrait of the five defined subgroups.

\section{RESULTS}

\section{Demographics and training}

Each subgroup has slightly more men than women, with a smaller percentage of women in the metabolic geneticist subgroup (Table 4). The median age for all geneticists is 52 years (data not shown), with adult geneticists having relatively larger numbers of younger geneticists. Because the overall survey did not include the most recent diplomates, the age distributions may be shifted upward toward older geneticists. Between $9 \%$ and $21 \%$ of geneticists in each subgroup report planning to retire in the next 5 years, with a higher number $(21 \%)$ reported by metabolic geneticists.

Approximately $90 \%$ or more of the geneticists in each subgroup have taken MD clinical genetics training. Training in clinical genetic laboratory specialties varies across subgroups (Table 4 ). Of note, slightly more than half (54\%) of metabolic geneticists have training in clinical biochemical genetics.

Nearly all geneticists report training in other medical specialties, with pediatrics being the most common in three subgroups (general, pediatrics, and metabolic geneticists) (Table 4). Generally, the training is consistent with types of genetics patients seen.

\section{Primary work setting, work hours, professional activities, and income}

The most common work setting for geneticists in each subgroup is an AMC. In fact, AMCs are the sole work setting for almost the entire adult geneticist subgroup and for most pediatric and metabolic geneticists (Table 5). Physician practices are the next most common work setting, and approximately one-fourth of the reproductive and general geneticists work in these settings. However, few geneticists $(<10 \%)$ in any other subgroup report working in physician practices. The next most common setting, hospitals, is the worksite for small numbers of reproductive, general, and pediatric geneticists. 
Table 4

Geneticist subgroups: Demographic characteristics, retirement plans, and training

\begin{tabular}{|c|c|c|c|c|c|c|}
\hline & All & General & Pediatric & Metabolic & Reproductive & Adult \\
\hline \multicolumn{7}{|l|}{ Demographic characteristics $^{a}$} \\
\hline Women & $45 \%$ & $44 \%$ & $48 \%$ & $35 \%$ & $48 \%$ & $48 \%$ \\
\hline \multicolumn{7}{|l|}{ Age distribution } \\
\hline$<45$ y & 21 & 14 & 27 & 27 & 11 & 41 \\
\hline $45-54$ y & 47 & 49 & 38 & 40 & 65 & 45 \\
\hline$>54 \mathrm{y}$ & 32 & 37 & 35 & 33 & 24 & 14 \\
\hline Plan retirement in next $5 \mathrm{y}$ & 12 & 9 & 13 & 21 & 14 & 9 \\
\hline \multicolumn{7}{|l|}{ Genetics training $^{b}$} \\
\hline MD Clinical Genetics & 92 & 96 & 90 & 85 & 87 & 100 \\
\hline Clinical Biochemical Genetics & 17 & 9 & 19 & 54 & 4 & 14 \\
\hline Clinical Molecular Genetics & 9 & 6 & 9 & 15 & 5 & 21 \\
\hline Clinical Cytogenetics & 12 & 16 & 8 & 11 & 13 & 5 \\
\hline \multicolumn{7}{|l|}{ Other medical training ${ }^{b}$} \\
\hline Pediatrics & 75 & 87 & 89 & 92 & 35 & 23 \\
\hline Internal Medicine & 11 & 10 & 6 & 8 & 4 & 57 \\
\hline Obstetrics and Gynecology & 12 & 2 & 6 & 0 & 60 & 0 \\
\hline Other Specialties & 9 & 6 & 6 & 7 & 17 & 22 \\
\hline
\end{tabular}

${ }^{a}$ Rounding of values, may not total $100 \%$.

${ }^{b}$ May have taken more than one type of training in genetics or in other medical residencies.

Overall, geneticists' median weekly work hours vary from 52 to 60 hours with higher hours reported by reproductive and adult geneticists (Table 5). Geneticists in each subgroup reported working approximately 48 weeks per year (median value, data not shown). The geneticists' work setting can play a major role in the ways that individual geneticists spend their professional time. For example, geneticists working as faculty/ clinicians in AMCs may be expected to spend time in several professional activities (e.g., patient care, teaching, research, and administration).

Patient care accounts for a large share of work hours for each subgroup. Median weekly patient care hours vary across subgroups from 15 to 33 hours with higher numbers of hours reported by reproductive geneticists and lower numbers of hours reported by adult and metabolic geneticists.

In three subgroups, between $62 \%$ and $76 \%$ of geneticists report spending 20 hours or more per week in patient care (reproductive, general, and pediatric) (Table 5). Slightly less than half of the metabolic geneticists and only one-third of adult geneticists report 20 hours or more in patient care. When analyzed by work setting (data not shown), large numbers of geneticists in physician practices $(87 \%)$ and hospital practice (76\%) report spending 20 hours or more with patient care. Smaller numbers of AMC-based geneticists (57\%) or geneticists in other settings (48\%) spend 20 hours or more per week with patient care.

Time not spent in patient care is spent in other professional activities; thus, there is a reciprocal relationship between pa- tient care hours and nonpatient care hours, although the latter include many activities. For this analysis, we define a substantial time commitment to any activity as at least $15 \%$ of a workweek (or 8-10 hours per week) (Table 5).

With this criterion, relatively few geneticists report substantial time spent in clinical laboratory work. Somewhat more geneticists (particularly adult, metabolic, and pediatrics geneticists) report substantial time in research (basic science or clinical research). In the work-setting analysis, approximately onequarter of geneticists working in AMCs or in other settings report substantial time in each research area, with few geneticists $(<5 \%)$ in either physician or hospital practice settings reporting time in research (data not shown).

Teaching is a substantial activity for approximately $20 \%$ of geneticists in the pediatric, general, and reproductive geneticist subgroups (Table 5). However, many geneticists in each subgroup report some time ( $1 \%-14 \%$ of total work time) in teaching (data not shown). By work setting (data not shown), 24\% of geneticists working in AMCs reported substantial teaching time with few geneticists working in other settings (10\%), hospitals (4\%), or physician practices (2\%) reporting this activity.

Net professional income was less than $\$ 100,000$ for approximately $20 \%$ or more of geneticists in four of the five subgroups (Table 5). Reproductive geneticists had the highest income levels with more than one-third earning more than $\$ 200,000$ per year. Metabolic geneticists had the lowest income levels, with only $17 \%$ earning more than $\$ 150,000$ per year. Many factors not specifically assessed in this analysis can affect professional 
Table 5

Geneticist subgroups: Work setting, total and patient care work hours, distribution of work effort, and income

\begin{tabular}{|c|c|c|c|c|c|c|}
\hline & All & General & Pediatric & Metabolic & Reproductive & Adult \\
\hline \multicolumn{7}{|l|}{ Work setting } \\
\hline AMC & $68 \%$ & $59 \%$ & $80 \%$ & $75 \%$ & $48 \%$ & $96 \%$ \\
\hline Physician practice & 16 & 25 & 7 & 6 & 29 & 4 \\
\hline Hospital & 9 & 11 & 8 & 5 & 17 & 0 \\
\hline Other setting & 6 & 6 & 5 & 14 & 5 & 0 \\
\hline \multicolumn{7}{|l|}{ Work hours $^{a}$} \\
\hline Total work hours/week & $55 \mathrm{~h}$ & $55 \mathrm{~h}$ & $52 \mathrm{~h}$ & $55 \mathrm{~h}$ & $60 \mathrm{~h}$ & $60 \mathrm{~h}$ \\
\hline Patient care hours/week & 27 & 28 & 26 & 19 & 33 & 15 \\
\hline \multicolumn{7}{|l|}{ Distribution of work effort } \\
\hline Patient care $>20 \mathrm{~h} / \mathrm{wk}$ & $63 \%$ & $71 \%$ & $62 \%$ & $46 \%$ & $76 \%$ & $33 \%$ \\
\hline Clinical laboratory $>15 \%$ time & 7 & 9 & 4 & 10 & 6 & 18 \\
\hline Basic science research $>15 \%$ time & 20 & 12 & 22 & 32 & 9 & 59 \\
\hline Clinical research $>15 \%$ time & 17 & 13 & 21 & 32 & 0 & 22 \\
\hline Teaching $>15 \%$ time & 18 & 21 & 22 & 8 & 20 & 0 \\
\hline Administration $>15 \%$ time & 16 & 21 & 11 & 11 & 15 & 30 \\
\hline \multicolumn{7}{|l|}{ Income } \\
\hline$<\$ 100,000$ & $22 \%$ & $21 \%$ & $28 \%$ & $29 \%$ & $7 \%$ & $18 \%$ \\
\hline$\$ 100,001-\$ 125,000$ & 21 & 22 & 22 & 33 & 10 & 16 \\
\hline$\$ 125,001-\$ 150,00$ & 20 & 23 & 20 & 22 & 10 & 25 \\
\hline$\$ 150,001-\$ 200,000$ & 22 & 25 & 20 & 10 & 23 & 33 \\
\hline$>\$ 200,000$ & 12 & 8 & 10 & 7 & 34 & 8 \\
\hline
\end{tabular}

AMC, academic medical center.

${ }^{a}$ Median work hours.

income or salary, including work setting, years in the job, and academic rank.

\section{Genetics patient care practice: Clinic staffing, referrals, and practice patterns}

The survey asked geneticists to describe the staffing at their primary genetics clinic. Because geneticists may attend many different clinics, the primary clinic was defined as the clinic where geneticists worked the most hours. Approximately half of all geneticists work in clinics where there are one or two geneticists (Table 6). This staffing pattern is especially common for reproductive geneticists, whereas other geneticists are more likely to have a higher geneticist staffing level.

Across all subgroups, most geneticists have one or more genetic counselors at their primary genetics clinic with many geneticists having three or more genetic counselors. Also, large numbers of geneticists in each subgroup report that genetic counselors see many or all of their new patients $(64 \%-77 \%$ of geneticists across all subgroups, data not shown).

Half of all geneticists report having nurses, either a registered nurse or a more highly trained nurse, at their primary genetics clinic. Nurses are more likely to be available in clinics with metabolic geneticists (75\%). Modest numbers of geneti- cists report that nurses who specialize in genetics see at least some of their patients. This practice is more common with metabolic $(31 \%)$ and pediatric $(24 \%)$ geneticists than other geneticists $(3 \%-6 \%)$ (data not shown).

Sources of new patient referrals are an important practice parameter. Most geneticists receive a majority of their referrals from other physicians, either generalist physicians (family medicine, internal medicine, pediatrics) or specialist physicians (including obstetricians and gynecologists) (Table 6). Patient self-referrals or family referrals account for many patient referrals for approximately $50 \%$ of geneticists in the adult geneticist subgroup and approximately $10 \%$ to $20 \%$ of geneticists in other subgroups. Other referral sources make up only a small portion of all referrals (e.g., managed care contracting, genetic testing laboratories, and genetic counselors).

Geneticists were asked to indicate how they spend their own time with new genetics patients. There was a striking similarity of time allocated to each of the six response activities as reported by each subgroup (data not shown). The following are the average (median) percentages of time in each patient care activity for all geneticists. Direct and "face-to-face contact with the patient and/or family" account for $66 \%$ of all patient care time. This is followed by "case research, data review, and con- 
Table 6

Geneticist subgroups: Genetics clinic staffing, referral sources, geneticists' new patient consultation visit patterns, and management of genetic and other medical conditions

\begin{tabular}{|c|c|c|c|c|c|c|}
\hline & All & General & Pediatric & Metabolic & Reproductive & Adult \\
\hline \multicolumn{7}{|l|}{ Genetics clinic staffing $^{a}$} \\
\hline \multicolumn{7}{|l|}{ Geneticists } \\
\hline One & $27 \%$ & $29 \%$ & $25 \%$ & $2 \%$ & $47 \%$ & $28 \%$ \\
\hline Two & 22 & 25 & 15 & 19 & 31 & 18 \\
\hline Three or more & 51 & 46 & 60 & 76 & 22 & 53 \\
\hline \multicolumn{7}{|l|}{ Genetic counselors } \\
\hline None & 14 & 16 & 11 & 10 & 19 & 19 \\
\hline One or two & 40 & 36 & 40 & 32 & 55 & 41 \\
\hline Three or more & 45 & 49 & 49 & 52 & 27 & 39 \\
\hline \multicolumn{7}{|l|}{ Nurses } \\
\hline None & 50 & 59 & 47 & 23 & 47 & 72 \\
\hline One or two & 37 & 32 & 39 & 51 & 38 & 28 \\
\hline Three or more & 12 & 8 & 14 & 24 & 15 & 0 \\
\hline \multicolumn{7}{|c|}{ Referral sources for many or all new patients ${ }^{a}$} \\
\hline Generalist physicians & 73 & 88 & 85 & 75 & 27 & 51 \\
\hline Specialist physicians & 68 & 66 & 57 & 68 & 91 & 77 \\
\hline Patient (self-referral or family) & 17 & 15 & 12 & 22 & 14 & 48 \\
\hline \multicolumn{7}{|c|}{ Geneticists' consultation pattern for many or all new patients ${ }^{a}$} \\
\hline One-time consult only & 65 & 66 & 58 & 52 & 84 & $<1$ \\
\hline Consult takes more than one visit & 40 & 44 & 41 & 55 & 16 & 38 \\
\hline \multicolumn{7}{|c|}{ Geneticists' ongoing management for many or all new patients ${ }^{a}$} \\
\hline Genetic condition managed & 38 & 32 & 45 & 72 & 8 & 30 \\
\hline Other conditions managed & 25 & 21 & 24 & 52 & 14 & 15 \\
\hline
\end{tabular}

${ }^{a}$ Categoric responses about many or all new patients do not total $100 \%$.

sultation notes" ( $15 \%$ of time), "other communication with the patient or family" (6\%), "communication with other genetics professionals" (5\%) or "with referring physicians" (6\%), and "administrative tasks related to clinical care" (5\%). The similarity of responses from each subgroup suggests that the genetic evaluation of new patients has a professional pattern and time allocation that is similar across each subgroup of geneticists.

A majority of patients seen by geneticists each year are new patients, referred for genetic evaluation. Geneticists may require one or more visits to complete a genetic evaluation. Approximately two-thirds of all geneticists report that their referral consultations for many or all new patients can be completed in one visit. A single-visit consultation pattern is most common for reproductive geneticists, slightly less common for other subgroups, and almost never the practice for adult geneticists (Table 6).

The geneticist, the patient's primary care physician, or other medical or surgical specialists may provide the ongoing management of many of the patients referred to geneticists. Less than half of all geneticists report that they provide ongoing management of the genetic condition for many or all patients. Providing ongoing management of the genetic condition for many or all new patients is a more common practice for metabolic $(72 \%)$ and pediatric (45\%) geneticists (Table 6). Fewer geneticists provide ongoing management of other medical conditions for many or all patients. Only metabolic geneticists report a high participation rate (52\%) with the management of other conditions for many or all patients; only $14 \%$ to $24 \%$ of other geneticists report this.

\section{Patient care visits and capacity to expand the practice}

The total visits per week (new and follow-up visits) for each clinical subgroup varies from 5 to 16 patients (Table 7). New patient visits outnumber follow-up visits for all subgroups except metabolic geneticists. The annual patient care productivity data show differences across subgroups. For each subgroup, the mean total visits are higher than the median values, indicating the number of visits is skewed toward the upper end. In 
Table 7

Genetic patient care: Hours and visits per week, annual genetic patient care productivity, capacity to see new patients, and change in patient volume over last three years

\begin{tabular}{|c|c|c|c|c|c|c|}
\hline & All & General & Pediatric & Metabolic & Reproductive & Adult \\
\hline \multicolumn{7}{|l|}{ Patient visits per week } \\
\hline New patient visits/week & 6 & 7 & 6 & 3 & 11 & 3 \\
\hline Time spent with new patient & $65 \mathrm{~min}$ & 75 & 75 & 90 & 60 & 70 \\
\hline Follow-up patient visits/week & 4 & 5 & 4 & 5 & 5 & 2 \\
\hline Time spent with follow-up patient & $40 \mathrm{~min}$ & 45 & 45 & 30 & 30 & 30 \\
\hline Patient care hours/week ${ }^{a}$ & $27 \mathrm{~h}$ & $28 \mathrm{~h}$ & $26 \mathrm{~h}$ & $19 \mathrm{~h}$ & $33 \mathrm{~h}$ & $15 \mathrm{~h}$ \\
\hline \multicolumn{7}{|l|}{ Annual patient care productivity ${ }^{b}$} \\
\hline New patients/year (median) & 288 & 336 & 288 & 156 & 523 & 147 \\
\hline New patients/year (mean) (SD) & $407(504)$ & $365(238)$ & $325(276)$ & $222(187)$ & $901(523)$ & $249(325)$ \\
\hline Follow-up patients/year (median) & 200 & 215 & 208 & 245 & 222 & 100 \\
\hline Follow-up patients/year (mean) (SD) & $314(338)$ & $307(319)$ & $280(235)$ & $384(449)$ & $405(437)$ & $155(180)$ \\
\hline Total patients/year (median $)^{b}$ & 539 & 576 & 510 & 400 & 1,043 & 255 \\
\hline Total patients/year (mean)(SD) & $723(690)$ & $673(443)$ & $606(469)$ & $612(602)$ & $1,310(1,202)$ & $405(437)$ \\
\hline \multicolumn{7}{|l|}{ Wait time for new patient appointment ${ }^{c}$} \\
\hline $1 \mathrm{wk}$ & $14 \%$ & $10 \%$ & $5 \%$ & $2 \%$ & $54 \%$ & $5 \%$ \\
\hline $2-3 \mathrm{wk}$ & 38 & 43 & 31 & 41 & 40 & 36 \\
\hline $1-3$ mo & 36 & 38 & 50 & 32 & 6 & 53 \\
\hline$>3 \mathrm{mo}$ & 11 & 10 & 12 & 23 & 0 & 6 \\
\hline \multicolumn{7}{|l|}{ Ability to accept new patients ${ }^{c}$} \\
\hline Practice full: cannot accept new patients & $5 \%$ & $6 \%$ & $2 \%$ & $12 \%$ & $6 \%$ & $0 \%$ \\
\hline Practice almost full: can accept some patients & 63 & 60 & 68 & 67 & 50 & 77 \\
\hline Practice far from full: can accept many new patients & 32 & 35 & 30 & 21 & 43 & 23 \\
\hline \multicolumn{7}{|l|}{ Change in patient volume in past 3 years } \\
\hline Decreased & $12 \%$ & $10 \%$ & $12 \%$ & $5 \%$ & $23 \%$ & $12 \%$ \\
\hline Stayed the same & 29 & 29 & 27 & 29 & 30 & 22 \\
\hline Increased & 43 & 47 & 44 & 45 & 31 & 46 \\
\hline Increased significantly & 16 & 14 & 17 & 21 & 16 & 20 \\
\hline
\end{tabular}

SD, standard deviation.

${ }^{a}$ Median values.

${ }^{b}$ Total visits calculated at individual level and median value reported; cannot sum median values to obtain total.

${ }^{c}$ Rounding of values, may not total $100 \%$.

addition, the standard deviation of the distribution of visits is high, indicating a wide range of reported visits (Table 7).

All data show a consistent pattern that reproductive geneticists provide from two or three times the number of visits per year as geneticists in other subgroups. The general and pediatric geneticists provide higher numbers of visits than the other two subgroups (metabolic and adult geneticists).

The survey collected information on measurable factors that can affect total patient care productivity. Total patient care hours vary across subgroups and show that geneticists who spend more time with patient care report seeing more patients.
All subgroups report lengthy patient visits, from 60 to $90 \mathrm{~min}$ utes for new patient visits and 30 to 45 minutes for follow-up visits. The time spent with patients varies across subgroups, with follow-up visits taking between $30 \%$ and $60 \%$ of the time spent with new patient visits.

The ability of geneticists to expand their practice by seeing additional new patients was assessed using responses from two survey questions. The first asked about the typical waiting time for scheduling nonurgent, new patients. The second asked the geneticists to directly assess whether their practice could accommodate additional new patients (Table 7). 
The reproductive geneticists stand out for reporting the capacity to see many new patients within a short time frame (1-3 weeks). More than half of other geneticists report a waiting time of 1 to 3 months or longer to schedule a nonemergent new patient. Because many patients referred to reproductive geneticists are pregnant, the time frame for an appointment is critical.

Few geneticists report that their practice is full, although approximately two-thirds in each subgroup report that their practice is almost full and they can accept only some new patients. On the other hand, approximately $20 \%$ to $40 \%$ of all geneticists report their practice is far from full and many new patients can be accepted.

Geneticists were asked to indicate whether their patient volume had changed in the past 3 years (Table 7). Approximately $30 \%$ of geneticists in each subgroup report stable volume, whereas $60 \%$ of each subgroup (except reproductive geneticists) report increases in patient volume. Approximately $10 \%$ of practices saw decreases in volume, with approximately $25 \%$ of reproductive geneticists reporting this was the case.

\section{DISCUSSION}

This study represents the first formal attempt to identify clinically relevant subgroups of geneticists with an evidencebased approach. With the use of survey data, we identified five subgroups of geneticists (general, pediatric, metabolic, reproductive, and adult geneticists) on the basis of the most pronounced type (s) of genetics patients in each geneticist's overall patient mix.

The subgroup classification criteria should be considered as necessary tools for the analysis, but not absolute criteria to define individual geneticists. Of interest, several of the interviewed clinical geneticists from the national genetics study referred to themselves as a "pediatric geneticist" or "practicing as a general geneticist." 7 Most of the geneticists we interviewed could be classified into one of the subgroups on the basis of the qualitative data they provided.

\section{Clinical genetics practice}

The subgroup analysis describes patterns of training, professional activities, and genetics practice. For example, reproductive geneticists have the most diverse work settings, with approximately half working in non-AMC settings such as physician practices and hospitals (Table 5). Metabolic geneticists rarely work as the only geneticist at their primary clinic, although they often work with a nurse, and they provide ongoing management to their patients (Table 6). General geneticists and pediatric geneticists share many characteristics, including diversity of patients, training, genetics clinic staffing, patient care hours, and patient care productivity (Tables 3, 4, 6, and 7). Greater numbers of general geneticists report seeing some adult and reproductive patients and working in nonAMC settings (Tables 3 and 5).

Because of the nature of geneticists' professional practice, many questions and responses are interrelated; thus one would expect to see relationships between variables. For example, ge- neticists who spend more time in research and teaching will have less time for direct patient care. One would expect geneticists recruited to work at AMCs to have career plans for research and to allocate time to this activity. This is seen with adult geneticists; almost all work in AMCs and many spend substantial time in research and clinical laboratories (Table 5). Also, adult geneticists are the smallest subgroup and they have the fewest patient visits and fewest hours spent in patient care (Tables 1 and 7). The patient visit data also may reflect the current lower demand for genetics referrals for adult patients. Currently, pediatric patients make up $76 \%$ of all new patient referrals to geneticists. ${ }^{5}$ However, adult and cancer genetics are emerging fields, and this may increase future referrals to adult geneticists. Of note, each of these survey findings were supported by clinical geneticists from various settings interviewed in the national study. ${ }^{7}$

\section{Patient care productivity}

The patient care productivity or number of patients whom a clinical geneticist sees annually varies by clinical subgroup. As noted, some of this variation is explained by measurable factors such as total time spent in patient care or the number of genetic counselors available to assist geneticists. However, other nonmeasured factors can also affect productivity. These include patient specific factors such as the complexity of the genetics problem, the available information, and the communication skills of the patient. Also, individual geneticists may differ in the amount of time they routinely allocate to each patient or their overall efficiency with seeing patients.

In addition, from the interviews we learned of the importance of the clinical setting in facilitating or hindering a geneticist's patient care productivity. Geneticists mentioned several factors that could increase their own productivity, including improvements in overall patient processing, efforts to reduce no-show patients, the presence of genetic counselors, and various outreach efforts to other physicians to increase the number of referrals. ${ }^{7}$

Another way to assess geneticists' patient care productivity is to compare geneticists with other medical specialists. This method is imperfect because of variations in models of practice across medical specialties and major data limitations. However, a comparative analysis can offer useful information for geneticist workforce planning.

The 2001 AMA surveys of selected specialties show the following for total work hours and patient care hours (median values): pediatricians ( 53 hours, 47 hours, respectively), internists (60 hours, 54 hours, respectively), obstetricians and gynecologists ( 57 hours, 53 hours, respectively), and neurologists (58 hours, 51 hours, respectively). ${ }^{11}$ Among geneticists who report working 20 hours or more in patient care, the total work hours and patient care hours are 55 hours and 36 hours respectively. Thus, geneticists report total work hours that are similar to other specialties, but patient care hours are much lower.

Geneticists provide far fewer total patient visits than other physicians, although this is almost entirely because of the small number of follow-up visits. In fact, geneticists 
provide approximately the same number of new visits per week as other physicians. The comparative data include total weekly visits in all settings, total weekly office visits, and new patient weekly office visits (all median values) as follows: pediatricians $(102,100$, and 8 , respectively), internists (100, 64, and 6, respectively), obstetricians and gynecologists $(97,75$, and 10, respectively), and neurologists (75, 50, and 15 , respectively). ${ }^{12}$ Comparable numbers for geneticists working 20 hours or more in patient care are 14 total visits and 8 new patient visits (data not shown). For all study geneticists these numbers are 10 total visits and 6 new patient visits per week (Table 7). Thus, the difference in total visits is the result of the low number of follow-up visits for geneticists (4-6 visits per week) compared with the high follow-up visit number for other medical specialties (35-90 visits per week).

The small numbers of follow-up visits to geneticists are consistent with the survey data on geneticists' practice patterns. Many geneticists report they complete a consultation in one visit and relatively few provide ongoing management for many or all of their patients. The comparative physician data include a large number of physicians in private practice and relatively fewer physicians employed in AMCs or other settings. Employment characteristics do have an effect on patient care productivity for other physicians, with generally higher patient visits reported by self-employed physicians. ${ }^{6}$

\section{Study limitations}

There are two major limitations in this study. The first involves data limits related to the survey sample. Because the original survey did not include the most recently certified geneticists (certified in 2002/2003 or 2005), the newer (and younger) geneticists are not reflected in the survey sample. The subgroups vary in size, with the adult geneticist subgroup having only minimally sufficient numbers for analysis. The general constancy of findings between the interviews and survey data are noteworthy, although the interview sample was small and not intended to be representative of all geneticists.

The second limitation relates to the analysis method, specifically the decision to use descriptive analyses rather than more complex statistical analysis. We chose to present descriptive analyses to provide a large amount of information, mostly in tabular form to allow readers to use the data for various questions of interest. The data are sufficient to allow for statistical analysis of differences among subgroups and the ways that variables correlate with each other. Of even greater interest would be to assess how specific variables (workplace setting, subgroup type) affect key outcomes of interest (e.g., patient care productivity) when controlling for other variables (e.g., patient care time). However, these analyses can be conducted in future studies.

\section{Implications for genetics training and workforce planning}

This study supports a recommendation that medical genetics workforce planning efforts consider clinical geneticist subgroup practice patterns and characteristics. However, this rec- ommendation is tempered by the reality that the total number of clinical geneticists is modest, and the geographic distribution favors states with large metropolitan areas and AMCs. ${ }^{3}$ In large metropolitan areas, clinical subgroup level planning could be considered. In locations where geneticists are few, such as rural states, planning should take into account the likelihood that these geneticists will need to be generalists to meet population needs.

Many practicing geneticists report limited ability to expand their current patient care capacity. This indicates that additional geneticists or increased numbers of other genetic service providers will be needed to meet any substantial growth in demand for genetic services. Although individual geneticists may achieve incremental expansion in patient care productivity, because of the small number of all clinical geneticists, it is unlikely that marginal efficiency gains will be able to accommodate large increases in new patient demand.

Several findings suggest that metabolic geneticists have the most limited ability to expand their practices and that this situation may get worse over time. Approximately one-fourth of metabolic geneticists have new patient waiting times of more than 3 months. Approximately three-fourths report that their practices are full or nearly full, and approximately $20 \%$ of metabolic geneticists report planning to retire in the next 5 years (Table 5 and 7).

Finally, workforce planning for clinical geneticists should take into account the role of other genetics professional groups who provide direct genetics patient care services, notably genetic counselors and advanced practice nurses in genetics. ${ }^{13,14}$ A key unknown is the extent to which other medical specialists will provide genetic services for conditions within their specialty (e.g., neurologists, maternal fetal medicine obstetricians, and others). Further research is needed on the scale and scope of genetic services provided by other medical specialists. Thus we continue to suggest that genetics workforce planning should not be carried out in isolation and should engage a broadly representative group that includes medical geneticists, genetic counselors, advanced practice nurses in genetics, and genetics-savvy physicians in other medical specialties and primary care. ${ }^{3}$

\section{ACKNOWLEDGMENTS}

Funding for the national genetic services workforce study supported the survey design, data collection, and preliminary analyses of the survey data. This funding was provided to the University of Maryland through a cooperative agreement (U78 HP 00016-01) with the Health Resources and Services Administration Bureau of Health Professions and Maternal Child Health Bureau Genetics Services Branch, and the National Institutes of Health National Human Genome Research Institute and Ethical, Legal, and Social Implications Program. Funding for this subgroup analysis was supported by Agreement No. 1 U22MC03957-01 between the ACMG and the University of Maryland in collaboration with National Coordinating Center for Regional Genetics and Newborn Screening Collaborative Groups, a cooperative agreement awarded to the ACMG by the 
Genetics Services Branch/ Maternal Child Health Bureau/ Health Resources and Services Administration. The University of Maryland School of Medicine and ACMG also supported staff time. We acknowledge the helpful suggestions received from reviewers. Other members of the research team contributed to our overall understanding of genetic services and the analysis of data.

\section{References}

1. American Board of Medical Genetics (ABMG). 2005 Numbers of Certified Specialists in Genetics. http://www.abmg.org/genetics/abmg/stats-allyears.htm. Accessed June 15, 2006.

2. American Board of Medical Specialties (ABMS). The ABMS Annual Report and Reference Handbook, 2005. http://www.abms.org/Downloads/Publications/AnnualReport2005. pdf. Accessed June 15, 2006.

3. Genetic Health Services Research Center. The Medical Geneticist Workforce: 2003 Survey of ABMG Certified Geneticists. http://medschool.umaryland.edu/ghsrc. Accessed June 15, 2006.

4. Korf BR, Feldman G, Wiesner GL. Report of Banbury Summit meeting on training of physicians in medical genetics, October 20-22, 2004. Genet Med 2005;7:433-438.
5. Cooksey JA, Forte G, Benkendorf J, Blitzer M. The state of the medical geneticist workforce: findings of the 2003 survey of American Board of Medical Genetics certified geneticists. Genet Med 2005;7:439-443.

6. Wassenaar JD, Thran SL, editors. Physician Socioeconomic Statistics, 2003 ed. Chicago: American Medical Association, 2003.

7. Genetic Health Services Research Center. Assessing genetic services and the health workforce. http://medschool.umaryland.edu/ghsrc/research_assessing.asp. Accessed June 15, 2006.

8. Center for Studying Health System Change: Design and methods for the community tracking study. http://hschange.org/index.cgi?data $=01$. Accessed June $15,2006$.

9. SSPS for Windows [computer program]. Release 11.0.0. Chicago: SPSS Inc., 2001.

10. Gonzalez ML, editor. Physician Marketplace Statistics, 1995 ed. Chicago: American Medical Association, 1996.

11. Wassenaar JD, Thran SL, editors. Overview of the physician market. In: Physician Socioeconomic Statistics, 2003 ed. Chicago: American Medical Association, 2003: 34-36.

12. Wassenaar JD, Thran SL, editors. Overview of the physician market. In: Physician Socioeconomic Statistics, 2003 ed. Chicago: American Medical Association, 2003: $48-50$.

13. Lea DH, Williams JK, Cooksey JA, Flanagan PA, et al. U.S. genetics nurses in advanced practice. J Nurs Scholarship 2006;38:1-6.

14. American Board of Genetic Counseling (ABGC). 2005 ABGC Statistics for All Years. http://www.abgc.net/genetics/abgc/about/intro.shtml. Accessed June 15, 2006. 